\title{
A comparative study of smica in various body fluids of diagnosed cervical cancer patients and healthy women
}

\author{
Pooja A.Pachani, MBBS, MD¹, Rajendra R. Godbole, MD', Jeevitaa Kshersagar, M.Sc ${ }^{2}$, Rakhi Jagdale, MD², \\ Amita Gosavi, MD ${ }^{4}$, Somshekhar Patil, MD ${ }^{5}$, Rakesh k Sharma, MD¹, Meghnad G. Joshi, PhD²

\begin{abstract}
${ }^{1}$ Department of Obstetric and Gynecology, Dr. DY Patil Medical College, Hospital \& Research Center, ${ }^{2}$ Department of Stem Cells and Regenerative Medicine, DY Patil Education Society, Deemed University, Kolhapur; ${ }^{3}$ Department of Pathology, Shri Siddhivinayak Ganpati Cancer Hospital,

${ }^{4}$ Department of Preventive Oncology, Shri Siddhivinayak Ganpati Cancer Hospital, ${ }^{5}$ Department of Obstetrics and Gynaecology, Somshekhar Hospital, Miraj, India
\end{abstract}

\section{Objective}

Cervical cancer (CC) is a major public health problem in women, and its early detection can help reduce morbidity and mortality. The objective of this study was to compare serum levels of soluble major histocompatibility complex class I-related chain A (sMICA) levels in various body fluids between women diagnosed with CC and healthy women.

Methods

A case-control study was conducted at a tertiary care hospital and a cancer center in Kolhapur, India. Overall, 150 individuals (100 CC patients and 50 healthy women) participated after providing informed written consent. Demographic data, histopathology history, parity, and tumor, node, and metastasis (TNM) staging data were collected. Pap smears, saliva, blood, and urine samples were collected. Pap smears were examined microscopically, and sMICA levels in all samples were determined by enzyme-linked immunoassay (ELISA).

Results

The mean age of women with cervical cancer was $49.86 \pm 8.18$ years. Squamous cell carcinoma $(70 \%)$ was the most common histological variant in CC patients. Serum soluble sMICA levels differed significantly with parity and TNM staging $(P<0.05)$. Mean levels of sMICA were significantly different in samples (CC cases vs. healthy patients; saliva: $166.721 \pm 108.718$ vs. $0.039 \pm 0.005 \mathrm{pg} / \mathrm{mL}$; urine: $82.921 \pm 45.580$ vs. $0.010 \pm 0.005 \mathrm{pg} / \mathrm{mL}$; serum: $35.756 \pm 10.799$ vs. $0.039 \pm 0.005 \mathrm{pg} / \mathrm{mL}, P<0.001)$.

\section{Conclusion}

Levels of sMICA in body fluids can be considered as a diagnostic or prognostic tool to determine disease progression or tumor regression.

Keywords: Body fluids; Neoplastic processes; Parity; Papanicolaou test; Uterine cervical neoplasms

\section{Introduction}

Cervical cancer (CC) is a serious health concern of grave concern among women, and the global mortality rate is approximately $15.2 \%$ [1-3]. The rising burden of CC in India can be ascribed to moderate to poor living standards and to the predominance of human papilloma virus (HPV) in young women around 30 years of age [3]. CC cases have decreased in the urban population, but this is a concern in rural parts of India. The consequences can be seen in the form of changes in sexual activity $(67 \%)$, menopause $(56 \%)$, fatigue $(64 \%)$, bladder difficulties (54\%), and bowel difficulties (54\%) $[4,5]$.
Received: 2021.04.07. Revised: 2021.05.20. Accepted: 2021.10.13. Corresponding author: Meghnad G. Joshi, PhD

Department of Stem Cells and Regenerative Medicine, DY Patil

Education Society Deemed University, DY Patil Vidyanagar, Kasba Bawda, Kolhapur 416006, India

E-mail: drmeghnadjoshi@gmail.com

https://orcid.org/0000-0001-6060-244X

Articles published in Obstet Gynecol Sci are open-access, distributed under the terms of the Creative Commons Attribution Non-Commercial License (http://creativecommons org/licenses/by-nc/3.0/) which permits unrestricted non-commercial use, distribution, and reproduction in any medium, provided the original work is properly cited.

Copyright $\odot 2022$ Korean Society of Obstetrics and Gynecology 


\section{Obstetrics \& Gynecology Science}

Vol. 65 , No. 1, 2022

The present situation in rural India can be attributed to the illiteracy and ignorance of people. Despite the existing national guidelines for the examination of CC, the coverage of screening camps is very low $[6,7]$. The slow progressive nature of an illness over a time span of 10-20 years provides a window of diagnosis at an earlier stage and allows for prevention from the stage of mild dysplasia to cancer of the cervix [8]. Various screening techniques, such as visualization with Lugol's iodine and/or acetic acid, pap smears, and HPVdeoxyribonucleic acid testing, have been used as identification methods and can help to decrease the burden of the disease [9].

Although HPV is the primary cause of CC in women and is asymptomatic in many patients, its clinical diagnosis is often missed; later, these lesions progress to invasive CC. This invasion can be due to a faulty immune system, which does not clear the aberrant cells, and in turn develops evasive mechanisms, resulting in tumor growth. One of the interesting mechanisms is the downregulation of major histocompatibility complex class I chain-related molecule A (serum levels of soluble major histocompatibility complex class I-related chain A [sMICA], a cytotoxic T-cell and natural killer [NK] -cell ligand) on the surface of tumor cells $[10,11]$.

Many studies have shown that sMICA levels are higher in the serum of tumor epithelial cells and can be shed from tumor cell surfaces. Downregulation of SMICA plays a role in downregulating NK group member D (NKG2D), which activates the receptor, thus impairing the activity of NK cells and $T$ cells, especially cluster of differenciation $8(C D 8+) T$ cells. This causes a reduction in the immunogenic response, thereby reducing effective tumor rejection $[4,5]$. The contribution of sMICA to tumor characteristics and survival rates in CC patients is still being researched [12].

The study was carried out to compare and analyze sMICA levels in the body fluids of women diagnosed with CC and in their healthy counterparts. The objectives were to analyze the expression levels of sMICA in the cervical cytology, blood serum, saliva, and urine samples of normal women and in women diagnosed with CC as well as to compare CC patients and healthy women.

\section{Materials and methods}

A prospective case-control study was conducted at a tertiary care hospital and a cancer center between July 2018 and October 2020 in Kolhapur, India. Ethical clearance was obtained from the Institutional Ethical Committee. A purposive sampling technique was used in this study.

Patients with carcinoma in situ up to stage IV of the FIGO classification for carcinoma of the cervix as well as patients who had a histopathological report of carcinoma in situ up until Stage IV without any prior treatment were included. Inclusion criteria for the controls were women visiting the outpatient department (OPD) for spacing and women attending regular check-ups or who visited the OPD for the insertion of an intrauterine device.

Patients suffering from oral, breast, ovarian, or endometrial cancers, had any kind of infection, were lactating or pregnant, had immunosuppressive diseases or were on immunosuppressant drugs, had undergone treatment for carcinoma cervix, or who had been vaccinated against HPV were excluded. Women suffering from fibroids or endometriosis as well as those who had known cases of dysfunctional uterine bleeding, were pregnant or lactating, were suffering from a pelvic inflammatory disease, or had any kind of malignancies were excluded from being used as controls.

Sample size was calculated using the formula:

$$
n=2 \times\left[\frac{Z_{1}-\alpha_{12}+Z_{1}-\beta}{\mathrm{ES}}\right]^{2}
$$

where ' $n$ ' is the minimum sample size, ' $a$ ' is the selected level of significance, $Z_{1}-a / 2$ is the value from the standard normal distribution holding $1-\alpha / 2,1-\beta$ is the selected power, $Z_{1}-\beta$ is the value from the standard normal distribution holding 1- $\beta$ below it, and ES is the effect size. The minimum required sample size was 128 . Hence, a total of 150 individuals (100 CC patients and 50 healthy women) were recruited in the study after obtaining informed written consent.

Demographic data, histopathology history, parity, tumor, node, and metastasis (TNM) staging data were recorded. Pap smear, blood, and body fluid samples were collected at the first visits of each of the patients after ensuring that the patients had not taken any prior treatments in any form (for example, radiotherapy, chemotherapy, or surgical interventions).

Informed consent was obtained from all patients. Patients $(n=100)$ who underwent elective surgical resection of histologically proven CC between 2015-2018 were included in the study. The tumor tissues were snap-frozen in liquid nitrogen immediately and placed in a freezer for further use. 


\section{Obstetrics \& Gynecology Science}

Pooja A.Pachani, et al. DX of carcinoma cervix with sMICA

The follow-up data of these patients were prospectively recorded for 6 months from the hospital. Paraffin-embedded tissue blocks from the same patients were obtained from the Department of Pathology for immunohistochemistry (IHC) analysis. Retrospective colon cancer tissue biopsies were used for comparative analysis.

\section{Preparation of body fluids}

\section{1) Saliva samples}

The saliva samples were collected in the morning. The patients refrained from eating food, drinking water, or from cleaning their teeth one hour prior to salivary collection. Whole saliva was collected from the patients by spitting into a collecting cup without mechanical or chemical stimulation. The saliva samples were kept in an ice bucket at $0^{\circ} \mathrm{C}$ and then centrifuged at $12,000 \mathrm{rpm}$ for 10 minutes at $4^{\circ} \mathrm{C}$. The resulting supernatants were stored at $-80^{\circ} \mathrm{C}$ for further use.

\section{2) Blood samples}

Samples were taken for estimating SMICA concentration and were centrifuged, aliquoted, and stored at $-80^{\circ} \mathrm{C}$ for further use. Preoperative and postoperative blood samples of patients ( $n=100$ ) were collected during treatment for 6 months in order to determine sMICA levels. Serum samples from 50 age-matched healthy blood donors who were free from systematically screened abnormalities were collected as controls. Serum levels of sMICA were determined using sandwich enzyme-linked immunosorbent assay (ELISA) according to the manufacturer's instructions. Sera from the controls expressed low levels of sMICA, very close to the detection limit of ELISA.

\section{3) Urine samples}

Samples were collected from the participants in the morning (20 mL). Pooled urine samples were transported at $4^{\circ} \mathrm{C}$ and stored at $-80^{\circ} \mathrm{C}$ until further use.

4) Immunohistochemical staining analysis

Serial $5 \mu \mathrm{m}$ thick sections were obtained from formalin-fixed paraffin-embedded tissue blocks and transferred to positively charged glass slides (Pathnsitu Biotechnologies, Hyderabad, India). Colon cancer tissue biopsies were used as positive controls for the comparative analysis. Sections were deparaffinized, rehydrated with xylene, and successively graded with alcohol. Sections were incubated with mouse monoclonal IgG antibodies (Santa Cruz Biotechnology, Santa Cruz, CA, USA) diluted at 1:100 (v/v) and goat anti-mouse IgG-HRP conjugated antibodies (Santa Cruz Biotechnology). Visualization of MICA/B was achieved using 3,3-diaminobenzidine tetrahydrochloride (DAB Chromogen; BioGenex, Fremont, (A, USA). Coppery-colored cytoplasms and membranes of tumor cells were considered positive for MICA/B. Sections were counterstained and evaluated using a Nikon EclipsTi microscope (Tokyo, Japan).

Two investigators graded the expression of MICA/B in a blinded manner. MICA/B expression was graded as having weak $(+)$, moderate $(++)$, or intense $(+++)$ MICA/B signals. Negative MICA/B expression was defined as no MICA/B signal (-).

\section{5) Papanicolaou (PAP) smear}

The samples were collected from the cervical region on positively charged slides (PathnSitu Biotechnologies). Pap smears were fixed with chilled acetone-methanol (1:1), air-dried, and stored at $-40^{\circ} \mathrm{C}$. Before moving forward in processing, slides were washed with distilled water (D/W) containing $0.05 \%$ tween 20 , serum blocking was performed with $2 \%$ goat serum, and the slides were incubated with phycoerythrin conjugate MICA/B (Molecular Probes, USA; plates were coated with $5.6 \mu \mathrm{L} / \mathrm{mL}$ ) for one hour at room temperature, followed by a wash with D/W containing $0.05 \%$ Tween 20 . The mounting media used was DAKO fluorescent, and the slides were examined microscopically.

6) sMICA analysis of saliva, blood and urine samples Serum levels of sMICA $(\mathrm{pg} / \mathrm{mL})$ in pooled samples $(n=100)$ were measured by ELISA using a Duoset kit (R\&D Systems, Minneapolis, MN, USA). The ELISA plates were coated with the human MICA capture antibodies at $5.6 \mu \mathrm{L} / \mathrm{mL}$ in ELISA plate coating buffer overnight at room temperature, and then they were blocked using $1 \mathrm{X}$ reagent diluents. This was followed by incubation with the patient sera. Next, the plates were washed, aspirated, and incubated with the human MICA detection antibodies diluted in reagent diluents with $2 \%$ goat serum, washed again, and then incubated with streptavidin HRP A (5 $\mu \mathrm{L}$ in $1 \mathrm{~mL}$ reagent diluents). Finally, the plates were washed and developed using Substrates A and B. Finally, the plates were incubated with stop solution for 10 minutes. Absorbance was measured at $450 \mathrm{~nm}$ using a ELISA Microplate Reader (Jiangsu, China) according to the 


\section{Obstetrics \& Gynecology Science}

Vol. 65 , No. 1, 2022

manufacturer's instructions. In order to validate the sMICA levels in the saliva and urine samples of the CC patients and controls, saliva and urine samples were centrifuged. The supernatant was used for the incubation. The absorbance of sMICA concentrations in the saliva and urine samples was recorded at $450 \mathrm{~nm}$ and determined using a ELISA plus microplate reader according to the manufacturer's instructions as described above. sMICA levels $\geq 1 \mathrm{pg} / \mathrm{mL}$ were considered positive according to the detection limit of ELISA.

\section{7) Statistical analyses}

Data were analyzed using R software v 3.6.1. Mann-Whitney $\mathrm{U}$ tests and Kruskal-Wallis tests were used for comparisons between groups $(P<0.05)$.

\section{Results}

The participants who were diagnosed with CC in the study had a mean age of $49.86 \pm 8.18$ years.

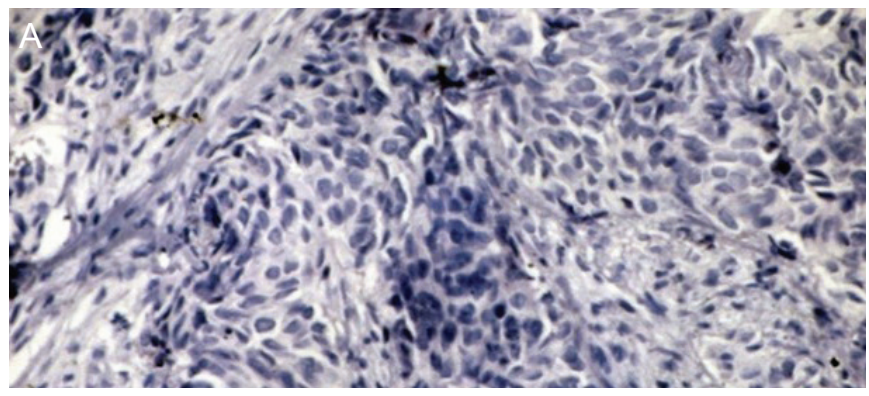

Control

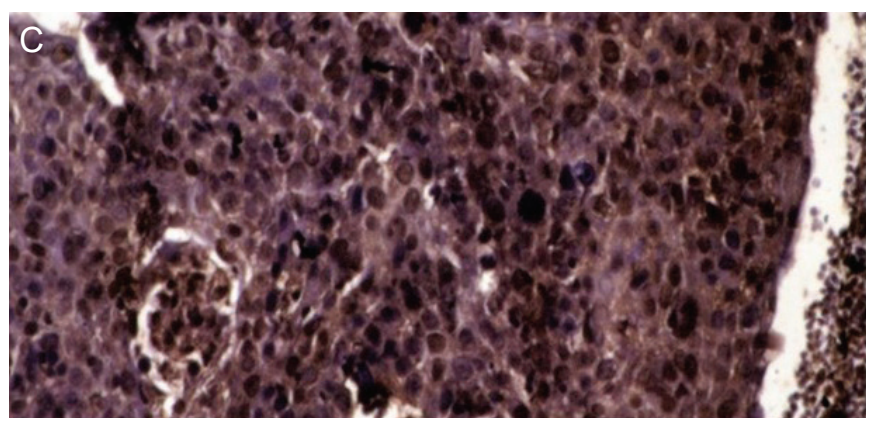

Stage II

\section{Tissue expression of MICA/B in CC}

MICA/B expression in tumor tissues was significantly increased compared to in non-tumor and non-stressed tissues. This demonstrates that 55 (87.3\%) of patients, which correlated with clinicopathological parameters characterized by early disease stages stage I $(n=33[58.73 \%])$ and II $(n=18[28.57 \%])$ with small, poorly differentiated tumors with lower invasion statuses and no lymphatic invasion (N0), expressed moderate MICA/B expression. In contrast, eight (12.69\%) MICA/B expression was low in the well-differentiated tumors that were $>4 \mathrm{~cm}$, had deeper invasion statuses, or were advanced TNM stage III tumors. Notably, patients with small poorly differentiated tumors at stages I and II show higher levels of MICA/B (Fig. 1) than the large welldifferentiated tumors at later disease stages III and IV.

\section{Soluble MICA (sMICA) in the sera of cervical cancer patients}

1) SMICA in the sera of CC patients

Our study evaluated MICA concentrations in the sera of cer-

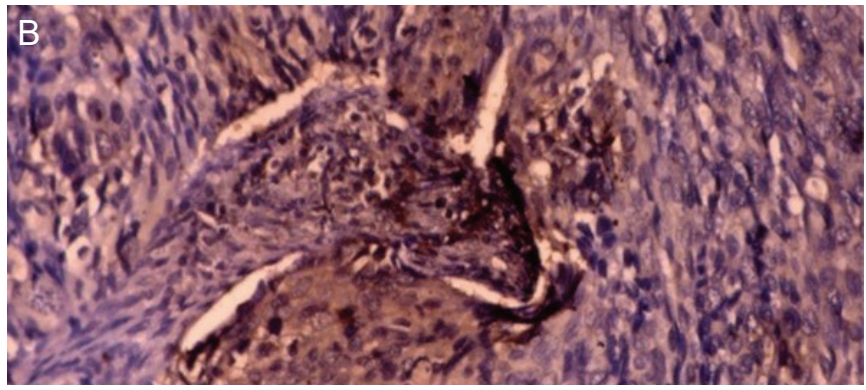

Stage I

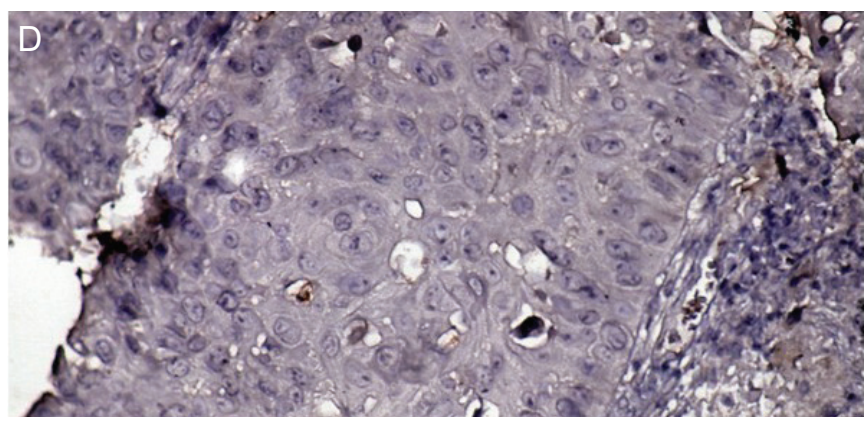

Stage III

Fig. 1. MICA/B expressions in different stages of oral cancer: MICA/B expression is higher in the tumor tissue than in control. (A) Representative IHC for MICA/B in control tissue sections. It did not show expressions of MICA/B (magnification $\times 40)$. $(B, C)$ IHC staining of poorly differentiated stage I and II tumor specimens showed intense to moderate staining for MICA/B and (magnification $\times 40)(D)$ well differentiated tumor specimens of stage III showed moderate staining (magnification $\times 40$ ). MICA/B, major histocompatibility complex class I chain-related protein A and B. 


\section{Obstetrics \& Gynecology Science}

Pooja A.Pachani, et al. DX of carcinoma cervix with sMICA

vical cancer patients to those of controls. We analyzed sMICA levels in the serum of CC patients enrolled in the study. The results revealed that the average levels of SMICA in CC patients were higher than those in controls. We assessed sMICA levels with respect to tumor size, grade of tumor differentiation, and disease stage. Tumor size and tumor stage are associated with sMICA levels; however, CC patients with stage IV disease has significantly higher serum levels of sMICA than those with stage I and II forms of the disease. The sMICA levels successively increased from stages I to III (Table 1). There were no significant differences in pre-and postoperative patient sera samples. It was also observed that sMICA concentrations in follow-up serum samples during treatment decreased in negligible amounts in CC patients (Fig. 2).

2) sMICA in the saliva and urine of CC patients In CC patients, sMICA levels in saliva and urine were increased in stage I, II, and III tumors, while sMICA levels in stage IV tumors were lower than in the other stages. Urine sMICA levels increased in stage III tumors and then decreased in stage IV tumors. The sMICA concentrations in the saliva samples of patients with cervical cancers in the different stages showed significant differences according to one-way analysis of variance ( $* * * * P<0.0001)$. As per Tukey's multiple comparisons test, sMICA concentration in saliva showed that all stages were significantly different, corresponding to each other, with $* * * * P<0.0001$. The sMICA concentration in saliva significantly increased at all stages (Fig. 3A). The sMICA concentration in urine samples of cervical cancer at different stages showed significant differences according to the oneway analysis of variance test $(* * * * P<0.0001)$. Tukey's multiple comparisons tests revealed that MICA concentrations in urine samples from all stages were significantly different from each other $(* * * * P<0.0001$; Fig. $3 B)$.

Overall, the total number of participants in the study was 150. In the case and control groups, the mean ages were

Table 1. Pre-treatment and post treatment levels of smica in the sera of cc patients and controls

\begin{tabular}{|c|c|c|c|c|c|c|c|c|c|}
\hline \multirow[t]{2}{*}{ Stage } & \multirow{2}{*}{$\begin{array}{l}\text { No. of } \\
\text { Patients }\end{array}$} & \multirow{2}{*}{$\begin{array}{l}\text { Pretreatment } \\
\text { patients } \\
\text { sMICA }(\mathrm{pg} / \mathrm{mL})\end{array}$} & \multicolumn{6}{|c|}{$\begin{array}{l}\text { Post treatment (F/W) } \\
\text { sMICA }(\mathrm{pg} / \mathrm{mL}) \text { Month }\end{array}$} & \multirow{2}{*}{$\begin{array}{c}\text { sMICA } \\
\text { (pg/mL) } \\
\text { control }\end{array}$} \\
\hline & & & 1st & 2nd & 3rd & 4th & 5th & 6th & \\
\hline I & $35(35 \%)$ & $24.39 \pm 4.86$ & $24.04 \pm 0.71$ & $25.85 \pm 0.88$ & $26.53 \pm 0.39$ & $27.1 \pm 0.54$ & $27.36 \pm 0.33$ & $27.81 \pm 0.41$ & $\leq 1$ \\
\hline$\|$ & $31(31 \%)$ & $38.42 \pm 3.99$ & $38.44 \pm 0.52$ & $38.91 \pm 0.98$ & $38.49 \pm 0.43$ & $37.82 \pm 0.39$ & $37.61 \pm 0.38$ & $36.96 \pm 0.12$ & \\
\hline III & $28(28 \%)$ & $43.62 \pm 2.61$ & $41.57 \pm 0.33$ & $42.54 \pm 0.30$ & $42.3 \pm 0.22$ & $42.91 \pm 0.05$ & $43.26 \pm 0.34$ & $43.61 \pm 0.3$ & \\
\hline IV & $6(6 \%)$ & $57.58 \pm 2.99$ & $54.63 \pm 0.25$ & $55.23 \pm 0.30$ & $55.58 \pm 0.63$ & $56.18 \pm 0.43$ & $56.67 \pm 0.28$ & $57.12 \pm 0.12$ & \\
\hline
\end{tabular}

sMICA, serum levels of soluble major histocompatibility complex class I-related chain A. FW, follow-up.

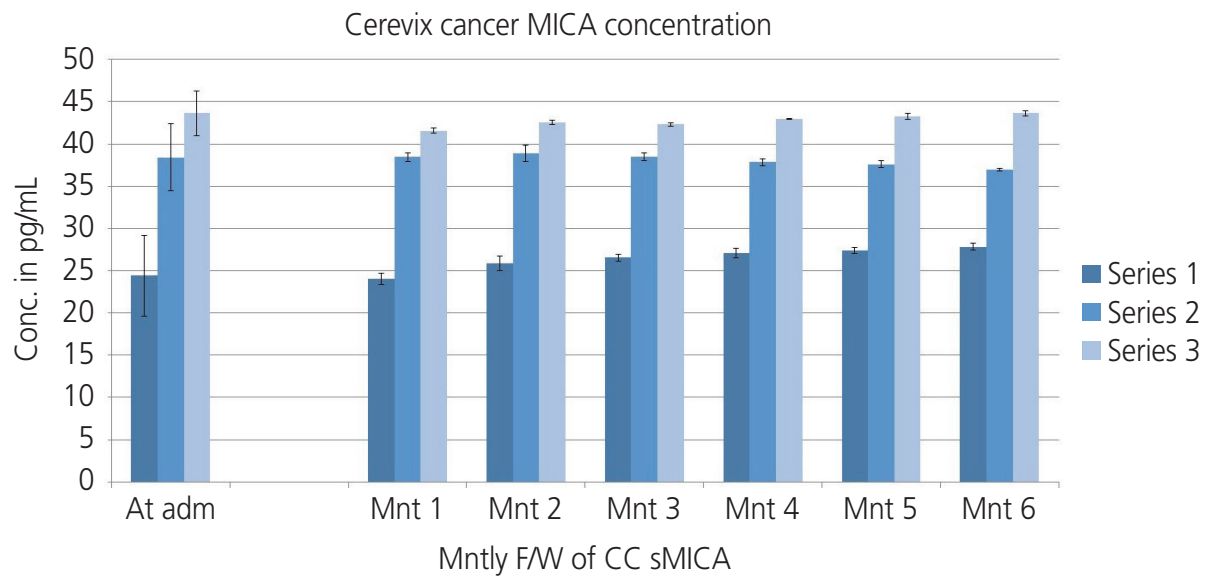

Fig. 2. Pre-treatment and post treatment levels of sMICA in the sera of CC patients and controls. MICA, major histocompatibility complex class I-related chain A; Conc., concentration; adm, admission; Mnt, Month; F/W, follow-up; sMICA, serum levels of soluble major histocompatibility complex class I-related chain A; CC, cervical cancer. 


\section{Obstetrics \& Gynecology Science}

Vol. 65, No. 1, 2022

$49.86 \pm 8.18$ years and $38.42 \pm 9.51$ years, respectively; the ages at the time of marriage in the case and control groups were $7.76 \pm 8.56$ years and $19.00 \pm 9.39$ years, respectively. A significant difference was observed in the mean age of the case group compared to the control group $(P<0.001)$. There was a statistically significant difference between the mean ages at marriage between carcinoma patients and healthy women $(P<0.001)$. In contrast, the histopathology in the control group was reported to be normal in most cases (40\%). (Fig. 4A). In terms of the histopathological examination results, the largest number of squamous carcinomas was observed in the case group (Fig. 4B).

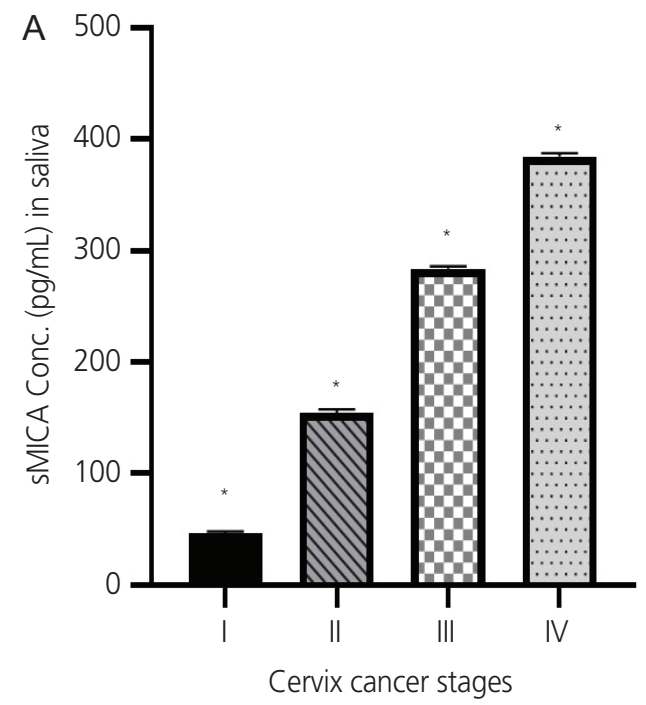

The levels of sMICA significantly differed within the same sample types with respect to parity $(P<0.05)$ (Table 2 ).

The levels of sMICA significantly differed within the same sample types with respect to the different TNM stages, and sMICA levels increased with each TNM stage $(P<0.001)$ (Table 3$)$.

The sMICA levels in the saliva, urine, and serum samples of the case group were as follows: $166.721 \pm 108.718$, $82.921 \pm 45.580$, and $35.756 \pm 10.799$, respectively. These values were $0.039 \pm 0.005,0.010 \pm 0.005$, and $0.039 \pm 0.005$, respectively, in the control group. The sMICA levels of the carcinoma patients significantly differed from those of the healthy women, as evidenced in the sample results: saliva

Fig. 3. (A) sMICA expression in the saliva of cervical cancer patients. (B) sMICA expression in the urine of cervical cancer patients. sMICA, serum levels of soluble major histocompatibility complex class I-related chain A. Conc., concentration. ${ }^{*} P<0.0001$.

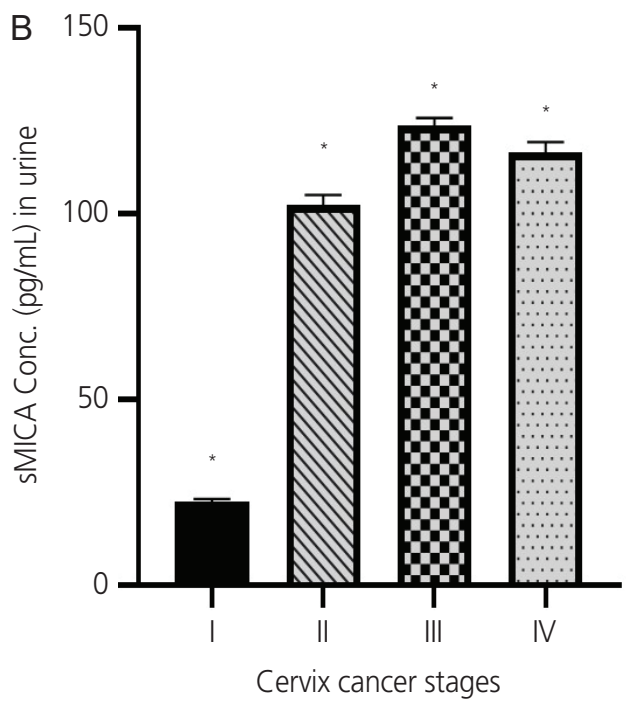

A

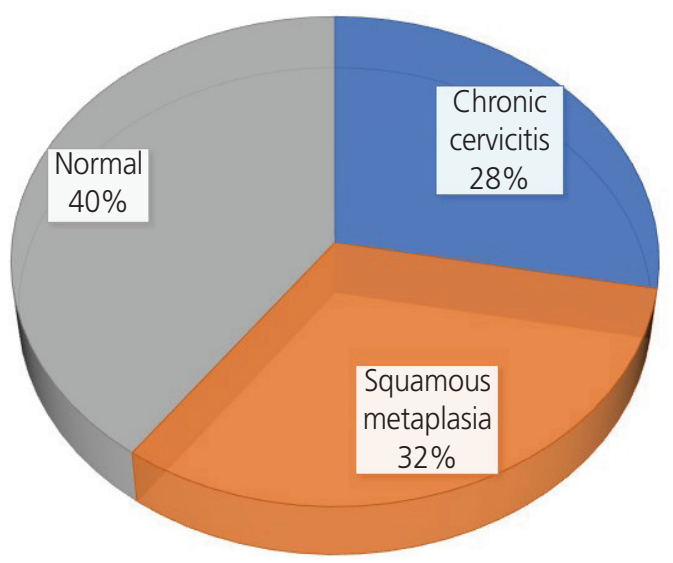

Fig. 4. (A) Histopathology distribution in healthy women. (B) Histopathology distribution in cervical cancer patients.

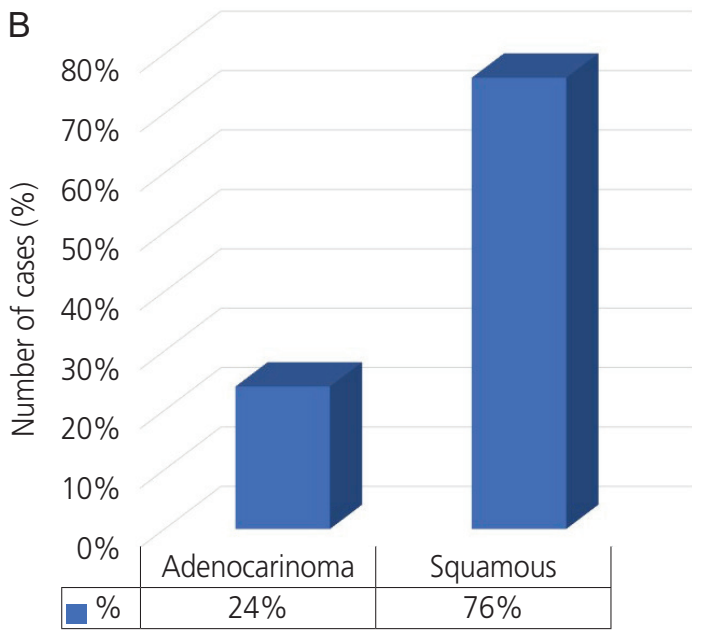




\section{Obstetrics \& Gynecology Science}

Pooja A.Pachani, et al. DX of carcinoma cervix with sMICA

$(P<0.001)$, urine $(P<0.001)$, and serum $(P<0.001)$.

\section{Discussion}

Cancer of the cervix is one of the most common malignancies in women, and its detection in earlier phases can help to lower mortality and morbidity [13]. Hence, screening for cervical cancer is of utmost importance so that it can be detected in earlier phases. Therefore, this study aimed to analyze sMICA levels in various body fluids of women diagnosed with CC compared to healthy women.

The mean age of the participants diagnosed with CC in the study was concurrent with the findings of Bobdey et al. [3], in which women diagnosed with carcinoma cervix were in the age range of $40-59$ years $(n=4,429 ; 53.7 \%)$. Hence, age can be considered a risk factor for carcinoma of the cervix

Table 2. Analysis of mean SMICA parameters by parity

\begin{tabular}{lccc}
\hline Parity & $\begin{array}{c}\text { Saliva } \\
(\mathbf{p g} / \mathbf{m L})\end{array}$ & $\begin{array}{c}\text { Urine } \\
(\mathbf{p g} / \mathbf{m L})\end{array}$ & $\begin{array}{c}\text { Serum } \\
(\mathbf{p g} / \mathbf{m L})\end{array}$ \\
\hline 0 & 29.22 & 14.99 & 15.01 \\
1 & 104.76 & 52.63 & 22.36 \\
2 & 92.6 & 45.31 & 18.92 \\
3 & 124.62 & 64.55 & 28.09 \\
4 & 176.59 & 86.31 & 35.04 \\
5 & 132.72 & 68.98 & 34.8 \\
6 & 284.74 & 123.23 & 44.52 \\
$P$-value & $0.0047^{\mathrm{a})}$ & $0.0041^{\mathrm{a})}$ & $0.0089^{\mathrm{a})}$ \\
\hline
\end{tabular}

sMICA, serum levels of soluble major histocompatibility complex class I-related chain A.

a) significant.

Table 3. Analysis of mean sMICA parameters by TNM stages

\begin{tabular}{lccc}
\hline TNM stage & $\begin{array}{c}\text { Saliva } \\
(\mathbf{p g} / \mathbf{m L})\end{array}$ & $\begin{array}{c}\text { Urine } \\
(\mathbf{p g} / \mathbf{m L})\end{array}$ & $\begin{array}{c}\text { Serum } \\
(\mathbf{p g} / \mathbf{m L})\end{array}$ \\
\hline $\mathrm{I}$ & 45.93 & 22.5 & 23.32 \\
$\mathrm{II}$ & 156.04 & 105.15 & 38.22 \\
$\mathrm{III}$ & 284.47 & 124.76 & 43.92 \\
$\mathrm{VI}$ & 377.04 & 125.26 & 57.48 \\
$P$-value & $<0.001^{\mathrm{a})}$ & $<0.001^{\mathrm{a})}$ & $<0.001^{\mathrm{a})}$ \\
\hline
\end{tabular}

sMICA, serum levels of soluble major histocompatibility complex class I-related chain A; TNM, tumor node and metastasis.

a) Significant, TNM, tumor, node, and metastases. because the risk of HPV infection increases with age [14].

The mean age at marriage of the participants who were diagnosed with carcinoma cervix was $27.76 \pm 8.56$ years. In contrast, in a study by Mhaske et al. [15], 86.4\% of women were married before 17 years of age. These contradictory findings can be attributed to the inclination of respondents in the present study to respond to questions in a way that can be viewed favorably by the researcher, which in turn gives rise to social desirability bias.

The most common histopathological variant in patients with CC was squamous carcinoma (70\%). Similar findings were reported by Wang et al. [16], in which squamous carcinoma accounted for $90 \%$ of CC cases.

Parity was identified as a risk factor, and histopathology has been considered a cornerstone for the identification of carcinoma of the cervix $[17,18]$. Hence, parity was used to recognize the histopathology of the study participants; however, it was observed that parity could not be used to identify the histopathology of the patients.

Many studies have shown that MICA levels can help predict the clinical outcomes of patients with cancer; however, this remains inconclusive [11]. Levels of serum-soluble sMICA significantly differed with respect to parity and TNM staging in this study $(P<0.05)$. In addition, a significant difference in the mean levels of sMICA was found in CC cases compared to healthy cases $(P<0.05)$. This difference directs the theory that SMICA is shed by tumor cells [11]. This shedding indicates a loss in binding capacity with its respective receptor molecules, which in turn leads to functional loss of the protein molecule and impacts the immune cells, especially the NK cells and CD8+T cells. Therefore, this leads to compromised capabilities of aberrant tumor cells [19].

Altogether, these results suggest that the increased expression of MICA/B in CC patients reflects persistent cell-associated expression of these NKG2DLs. In addition, tumor tissue expression of MICA/B favors NKG2D receptor downregulation in cytotoxic lymphocytes in the advanced stages. In the present study, we observed using $\mathrm{IHC}$ analysis that poorly differentiated tumors show higher levels of MICA/B than welldifferentiated tumors in the advanced stages.

The results indicate that SMICA levels varied between the cancer and control groups, with no correlations found with tumor size, grade, or survival rate. This study was limited by its small sample size. Further research without the aforementioned limitation as well as prospective trials are warranted. 


\section{Obstetrics \& Gynecology Science}

Vol. 65 , No. 1, 2022

The levels of sMICA in the body fluids of CC patients were higher than in healthy women. Therefore, this study suggests that sMICA levels in body fluids can be considered diagnostic or prognostic tools for which to analyze disease progression or tumor regression.

\section{Conflicts of interest}

The authors declare no conflicts of interest.

\section{Ethical approval}

This study does not require approval of the Institutional Review Board because no patient data is contained in this article. The study was performed in accordance with the principles of the Declaration of Helsinki.

\section{Patient consent}

Written informed consent and the use of images from patients are not required for the publication.

\section{Funding information}

Dr. Meghnad G. Joshi acknowledges the research funding support from the Department of Science and Technology (DST), Govt. of India (SB/SO/HS/0198/2013) and D.Y. Patil Education Society Deemed University (DYPES/DU/R\&D/3104).

\section{References}

1. Srivastava AN, Misra JS, Srivastava S, Das BC, Gupta S. Cervical cancer screening in rural India: status \& current concepts. Indian J Med Res 2018;148:687-96.

2. Arbyn $M$, Weiderpass $E$, Bruni L, de Sanjosé S, Saraiya M, Ferlay J, et al. Estimates of incidence and mortality of cervical cancer in 2018: a worldwide analysis. Lancet Glob Health 2020;8:e191-203.

3. Bobdey S, Sathwara J, Jain A, Balasubramaniam G. Burden of cervical cancer and role of screening in India.
Indian J Med Paediatr Oncol 2016;37:278-85.

4. Parish SJ, Nappi RE, Krychman ML, Kellogg-Spadt S, Simon JA, Goldstein JA, et al. Impact of vulvovaginal health on postmenopausal women: a review of surveys on symptoms of vulvovaginal atrophy. Int J Womens Health 2013;5:437-47.

5. Dalal PK, Agarwal M. Postmenopausal syndrome. Indian J Psychiatry 2015;57(Suppl 2):S222-32.

6. Catarino R, Petignat P, Dongui G, Vassilakos P. Cervical cancer screening in developing countries at a crossroad: emerging technologies and policy choices. World J Clin Oncol 2015;6:281-90.

7. WHO guidelines. WHO guidelines for screening and treatment of precancerous lesions for cervical cancer prevention. Geneva: World Health Organization; 2013.

8. World Health Organization. Comprehensive cervical cancer control: a guide to essential practice. 2nd ed. Geneva: World Health Organization; 2014.

9. Mishra GA, Pimple SA, Shastri SS. An overview of prevention and early detection of cervical cancers. Indian J Med Paediatr Oncol 2011;32:125-32.

10. Baranwal AK, Mehra NK. Major histocompatibility complex class i chain-related a (MICA) molecules: relevance in solid organ transplantation. Front Immunol 2017;8:182.

11. Zhao Y, Chen N, Yu Y, Zhou L, Niu C, Liu Y, et al. Prognostic value of MICA/B in cancers: a systematic review and meta-analysis. Oncotarget 2017;8:96384-95.

12. Samuels S, Ferns DM, Meijer D, van Straalen JP, Buist MR, Zijlmans HJ, et al. High levels of soluble MICA are significantly related to increased disease-free and disease-specific survival in patients with cervical adenocarcinoma. Tissue Antigens 2015;85:476-83.

13. Safaeian M, Solomon D, Castle PE. Cervical cancer prevention--cervical screening: science in evolution. Obstet Gynecol Clin North Am 2007;34:739-60, ix.

14. Sreedevi A, Javed R, Dinesh A. Epidemiology of cervical cancer with special focus on India. Int J Womens Health 2015;7:405-14.

15. Mhaske M, Jawadekar SJ, Saundale SG. Study of association of some risk factors \& cervical dysplasia/ cancer among rural women. Natl J Community Med 2011;2:209-12.

16. Wang W, Zhang F, Hu K, Hou X. Image-guided, intensity-modulated radiation therapy in definitive radiotherapy 


\section{Obstetrics \& Gynecology Science}

Pooja A.Pachani, et al. DX of carcinoma cervix with sMICA

for 1433 patients with cervical cancer. Gynecol Oncol 2018;151:444-8.

17. Kashyap N, Krishnan N, Kaur S, Ghai S. Risk factors of cervical cancer: a case-control study. Asia Pac J Oncol Nurs 2019;6:308-14.

18. Vinh-Hung V, Bourgain C, Vlastos G, Cserni G, De Rid- der M, Storme G, et al. Prognostic value of histopathology and trends in cervical cancer: a SEER population study. BMC Cancer 2007;7:164.

19. Schmiedel D, Mandelboim O. NKG2D ligands-critical targets for cancer immune escape and therapy. Front Immunol 2018;9:2040. 